\title{
Critical Components of Formative Assessment in Process-Oriented Guided Inquiry Learning for Online Labs
}

\author{
Saptarshi Purkayastha ${ }^{1}$, Asha K. Surapaneni ${ }^{1}$, Pallavi Maity ${ }^{1}$, Anushri S. Rajapuri ${ }^{1}$ and Judy \\ W. Gichoya ${ }^{2}$ \\ ${ }^{1}$ Indiana University - Purdue University Indianapolis, USA \\ ${ }^{2}$ Oregon Health Sciences University, USA \\ saptpurk@iupui.edu \\ asurapan@iu.edu \\ pmaity@iu.edu \\ anraja@iu.edu \\ gichoya@ohsu.edu \\ DOI: 10.34190/JEL.17.2.02
}

\begin{abstract}
In the traditional lab setting, it is reasonably straightforward to monitor student learning and provide ongoing feedback. Such formative assessments can help students identify their strengths and weaknesses, and assist faculty to recognize where students are struggling and address problems immediately. But in an online virtual lab setting, formative assessment has challenges that go beyond space-time synchrony of online classroom. As we see increased enrollment in online courses, learning science needs to address the problem of formative assessment in online laboratory sessions. We developed a student team learning monitor (STLM module) in an electronic health record system to measure student engagement and actualize the social constructivist approach of Process Oriented Guided Inquiry Learning (POGIL). Using iterative Plan-Do-Study-Act cycles in two undergraduate courses over a period of two years, we identified critical components that are required for online implementation of POGIL. We reviewed published research on POGIL classroom implementations for the last ten years and identified some common elements that affect learning gains. We present the critical components that are necessary for implementing POGIL in online lab settings, and refer to this as Cyber POGIL. Incorporating these critical components are required to determine when, how and the circumstances under which Cyber POGIL may be successfully implemented. We recommend that more online tools be developed for POGIL classrooms, which evolve from just providing synchronous communication to improved task monitoring and assistive feedback.
\end{abstract}

Keywords: POGIL, online education, formative assessment, health information management, electronic health records

\section{Introduction}

The 2018 report from the US National Center for Education Statistics showed that postsecondary enrollment dropped by almost 90,000, about 0.5\% lower between Fall 2016 to Fall 2017 (McFarland et al., 2018). On the other hand, students who were exclusively enrolled online grew by $0.7 \%$, up from $14.7 \%$ to $15.4 \%$ of all students. The proportion of all students who took at least one course online increased to $33.1 \%$. Thus, about a third of all postsecondary students are learning in an online setting. So as online education has gone mainstream, many researchers have argued for new pedagogies that are suitable for online education (Anderson \& Dron, 2011; Green et al., 2010; van Rooij \& Zirkle, 2016), including those for massive open online courses (MOOCs). As more students access online education, we also find that these students were less engaged compared to their peers from face-to-face classes (Dixson, Greenwell, Rogers-Stacy, Weister, \& Lauer, 2017; Friðriksdóttir, 2018). In online education, reconstruction of knowledge in a social constructivist view (Mingfei \& Jie, 2010) mainly through team-based learning, is harder to achieve in online courses, where spacetime factors separate learners and educators. Thus, many team or peer-based learning strategies such as Team-Based Learning (TBL) (Parmelee, Michaelsen, Cook, \& Hudes, 2012), Process-Oriented Guided Inquiry Learning (POGIL) (Moog, Spencer, \& Straumanis, 2006), Peer-Mediated Instruction (Utley \& And Others, 1997), Classwide Peer Tutoring (Delquadri, Greenwood, Whorton, Carta, \& Hall, 1986), Jigsaw technique (Perkins \& Saris, 2001), and a few more, require modifications for appropriateness in online settings. The Peer-Led Team Learning (PLTL) for example, is a pedagogy that has been successfully modified for online contexts, and with the modifications is referred to as Cyber PLTL or CPLTL (Janke \& Varma-Nelson, 2014; Mauser et al., 2011). In this paper, we present an empirical investigation of the social constructivist pedagogy called POGIL, identifying the critical components for POGIL in online lab sessions.

Reference this paper: Purkayastha, S. et al., 2019. Critical Components of Formative Assessment in Process-Oriented Guided Inquiry Learning for Online Labs. The Electronic Journal of e-Learning, 17(2), pp. 79-92, available online at www.ejel.org 
The BS in Health Information Management (HIM) undergraduate program at Indiana University-Purdue University Indianapolis (IUPUI) is a 68-year-old degree program and is now entirely online. HIM and Health Informatics are interdisciplinary fields which integrate biomedical sciences, information sciences, and computer science. We have employed a multitude of online engagement techniques: project-based learning and active learning strategies using virtual labs to engage students, but low engagement and lack of skills, observed when students enroll into graduate education, continues to be a serious issue. To solve the challenges of identifying online student engagement, being able to provide formative assessment and engage students with hands-on, team-based learning strategies in an online setting, we designed a novel monitoring tool called Student Team-Based Learning Monitor (STLM) (Purkayastha, Surapaneni, \& Maity, 2018) on OpenMRS, an open-source electronic health record (EHR) system. For the POGIL and STLM implementation we selected two courses from the HIM program for implementation, M200 - Database Design for HIM and M220 Health informatics for Decision Support. These two courses focus on students gaining process skills related to the design of electronic health record systems and database management systems for health care. The HIM M200 is also a general education course that is taken by students from different majors. Between the study periods of Spring 2017 and Fall 2018, students from 8 different majors had taken the course. Thus, we identify these courses and appropriate to identify the critical components, where hands-on labs play a critical role in students gaining process skills. The HIM field is moving towards broader use of technology and informatics (Bloomrosen \& Berner, 2017; Gibson, Dixon, \& Abrams, 2015), making it essential for students to have handson experience in using and innovating using technology. Thus, many education programs are in the transition to offer online courses with lab sessions, and our paper might be of interest to that audience.

To measure and track student engagement within the virtual lab setting, we developed the STLM tool in an electronic health record system and introduced in these two courses with the objective of monitoring students' activities and provide the efficient ways to complete a given task. Our main innovation of STLM has been presented elsewhere (Purkayastha, Surapaneni, et al., 2018). Features of this tool include capturing the user page navigation activity, onscreen annotations, SQL query execution, creating and exporting block/entity/workflow diagrams and comparison of task performed with other members of the group. These features were developed based on the POGIL activities designed for the two courses.

In this paper, we start by discussing the importance of formative assessment as a high-impact instructional practice. We then review published research on POGIL pedagogy in the last ten years, focusing on themes, critical components, course context and impact on student learning. We then present a contextual overview of our implementation of STLM and how it aids in the POGIL pedagogy. We empirically identify some of the critical components of POGIL which required adaptation due to the online setting, and how these were implemented into two HIM courses for three iterations of Plan-Do-Study-Act over a period of two years. We then describe the features of the STLM tool that helps to formative assessment in team-based knowledge construction, which is central to the POGIL approach.

\section{Formative assessment and critical components}

The use of early assessment to check if the learner understands things correctly is an age-old tradition in the practice of teaching, later conceptualized as formative assessment (Nicol \& Macfarlane-Dick, 2006; Sadler, $1989,1998)$. Proponents of formative assessment have often called it "a high-impact instructional practice," and as the single most effective intervention to increase student learning (Briggs, Ruiz-Primo, Furtak, Shepard, \& Yin, 2012). On the one hand, Black \& Wiliam (2009) developed a theory of formative assessment, but on the other hand, Bennett (2011) in the critical review of formative assessment says that the term does not have a well-defined set of artefact or practices. So, what do we understand by formative assessment? Formative assessment, for the purposes of this paper, is the process of teaching to recognize and respond to student learning in order to enhance it, during the process of learning (Bell \& Cowie, 2001; Cowie \& Bell, 1999). It involves both the planned as well as the interactive, just-in-time practices to interpret student learning and take action to enhance the learning. Planned formative assessment involves formulating the content in a way that students participate and express their understanding of concepts, which usually tends towards the class as a whole. Interactive formative assessment involves the teacher to notice, recognize and respond to an individual or groups of students. However, as classrooms have moved to online and blended learning settings, researchers have also reconceptualized formative assessment (Gikandi, Morrow, \& Davis, 2011). Techniques applied by individuals, peers, and teachers for formative assessment in these settings involve the use of online tools such as self-test quizzes, discussion forums and e-portfolio (Gikandi et al., 2011). 
A large part of the critique of the formative assessment has been its consideration as conventional wisdom in education, and the lack of empirical evidence that it increases learning gains (Dunn \& Mulvenon, 2009). But lately, formative assessment has started to gain traction with evidence in improving student engagement, and certain types of behavioral motivation is encouraging (Pereira, Flores, \& Niklasson, 2016). More so, there is a consensus that there is a harmful dichotomy that has developed between formative and summative assessment of teaching, and there needs to be an effort to link the two (Lau, 2016). This dichotomy needs to be understood as a fundamental challenge of assessing student learning, and if an early or complete understanding of concepts should be evaluated when assessing learning.

When we discuss and view online and blending learning, early assessment is challenging due to the differences in space-time between the teacher and the learner. Baleni (2015) articulated some of the pros and cons of formative assessment in online courses. The advantages include improvement in student commitment, faster feedback, enhanced flexibility, and the importance of procedure of taking tests and lectures resulted in less marking time and saved administration costs. On the other hand, the cons included trustworthiness and the lack of interaction in the formative feedback. However, these cons are something that could be dealt with innovation in e-learning tools, as well as processes for communication between the learners and the teachers. There is another challenge of teacher education in performing the formative assessment in the online learning environments. It is quite uncommon that teachers during their training receive formative assessment and thus, are unlikely to use it in their own teaching (Hamodi, López-Pastor, \& López-Pastor, 2017). There are also diverse formats through which teachers may provide online formative assessment such as multiple-choice quizzes, one-minute papers, e-portfolios, wikis, blogs, forums, mobile-based pop-quiz or student response systems (SRS), etc. (McLaughlin \& Yan, 2017). These are useful tools for interactive environments, particularly accompanying didactic lecture material, but do very little for lab sessions or courses that depend on hands-on work. More recently, researchers have developed tools that use artificial intelligence agents (Chattopadhyay, Shankar, Gangadhar, \& Kasinathan, 2018; Graesser \& McDaniel, 2017; Spector et al., 2016), semantic technologies (Santamaría Lancho, Hernández, Sánchez-Elvira Paniagua, Luzón Encabo, \& de Jorge-Botana, 2018) and online tutors (Dutton et al., 2017; Hooshyar et al., 2016; Lull \& Mathews, 2016). All of these suggest that there is an urgent need to support innovative tools that can help online assessment while students are doing online hands-on work, or learning in groups in an online environment.

There is a lingering question whether the implementation of formative assessment or some of the earlier pedagogy that we discussed has been done in a scientific way, which is appropriate to the original design of the pedagogy. Elsewhere, this has been referred to as fidelity of implementation (Stains \& Vickrey, 2017). The basis of Fidelity of Implementation (FIO) comes from implementation science and biomedical research, where it is essential that standards of procedure are followed, to be able to replicate drug studies and clinical trials. Offerdahl, McConnell, \& Boyer (2018) identified a set of critical constructs that can be thought of as a recipe through which pedagogy implementation can be verified to be true to its original intent. These critical constructs can help identify or differentiate an implementation of pedagogy from its original intent/theory.

\section{A review of process-oriented guided inquiry learning (POGIL)}

In the last couple of decades, there has been a shift, particularly in Science, Technology, Engineering and Mathematics (STEM) Education towards student-centered instructional techniques that are effective in achieving learning goals of the classroom. POGIL is one such instructional approach with a conscious emphasis on developing process skills and requiring distinctive classroom materials, which engage students in their own learning through the process skills. There are broadly three defining characteristics of the POGIL-specific material:

1. The material defines a POGIL activity to be used by self-managed teams that use the instructor as a facilitator of learning, rather than a source of information

2. The POGIL activity constructs knowledge among learners using exploration, refining and integrating shared concepts among the team of learners

3. The POGIL activity embeds in its structure and content the application and development of at least one targeted process skill, that may or may not necessarily be performed in the lab or the classroom. 
Even though POGIL started in Chemistry, its use and efficacy in improving learning have been shown in Medicine, Pharmacy, Computer Science, Math, Biology, Psychology and many other fields, in both secondary, higher secondary and university students. As POGIL inherits from guided inquiry, its core principles depend on a learning cycle of exploration, concept invention and application. POGIL was developed on the observations that (Hanson, 2006):

1. Most students don't learn through teaching by telling,

2. Students who are part of an interactive community are more likely to be successful, and

3. Knowledge is personal; students enjoy themselves more and develop greater ownership over the material when they are given an opportunity to construct their own understanding.

We searched for peer-reviewed articles in the English language on POGIL in the last ten years, with a focus on those studies which had analysis and data to demonstrate learning gains. We used Scopus as the database to look for articles and found a total of 112 articles. There seems to be a steady growing interest in POGIL-related publications - 6 (2010); 4 (2011); 12 (2012); 14 (2013); 11 (2014); 13 (2015); 13 (2016); 23 (2017); 16 (2018). However, most articles are introductory, some describe the pedagogy, some provide theoretical arguments and guidelines, and only a few have empirical implementations of POGIL. We found 33 such articles that showcased implementation of POGIL, of which seven papers were ignored because they were either reviews or were not peer-reviewed research articles. Three papers were dropped because even though they claimed to have POGIL implementation, they were incomplete or changed to another pedagogy mid-way. Five other papers did not clarify the POGIL activities or did not have enough information about how POGIL was implemented in the class, making it difficult to identify the critical components. We present a summary of the 18 papers in Table 1.

Table 1: A review of POGIL implementation themes and critical components

\begin{tabular}{|c|c|c|c|c|c|}
\hline & ARTICLE & THEME & $\begin{array}{c}\text { CRITICAL } \\
\text { COMPONENTS }\end{array}$ & CONTEXT & INSTRUMENTS \\
\hline 1. & $\begin{array}{l}\text { Smith, A. L., Paddock, J. R., } \\
\text { Vaughan, J. M., \& Parkin, D. } \\
\text { W. (2018). Promoting } \\
\text { Nursing Students' } \\
\text { Chemistry Success in a } \\
\text { Collegiate Active Learning } \\
\text { Environment: "If I Have } \\
\text { Hope, I Will Try Harder." } \\
\text { Journal of Chemical } \\
\text { Education, 95(11), 1929- } \\
\text { 1938. }\end{array}$ & $\begin{array}{l}\text { 1) To build hope in } \\
\text { students. } \\
\text { 2) To create self- } \\
\text { concept. } \\
\text { 3) Instructor was } \\
\text { having an open } \\
\text { attitude. }\end{array}$ & $\begin{array}{l}\text { POGIL } \\
\text { implementation: } \\
\text { Small group work } \\
\text { In-class POGIL } \\
\text { activities. } \\
\text { 2) The strategic } \\
\text { organization and } \\
\text { alignment of } \\
\text { learning } \\
\text { outcomes. } \\
\text { 3) A decidedly } \\
\text { active learning } \\
\text { environment. } \\
\text { 4) Health-related } \\
\text { scenarios. }\end{array}$ & $\begin{array}{l}\text { 1) In-Class } \\
\text { 2) Undergraduate, } \\
\text { one-semester } \\
\text { course, mostly } \\
\text { nursing students. } \\
\text { 3) } N=40\end{array}$ & $\begin{array}{l}\text { 1) CSCI } \\
\text { (Chemistry Self- } \\
\text { Concept } \\
\text { Inventory) } \\
\text { 2) SALG data was } \\
\text { collected from } \\
2008-2010 \text { and } \\
2010-2016 \text {. } \\
\text { 3) Course } \\
\text { assessment }\end{array}$ \\
\hline 2. & $\begin{array}{l}\text { Saputro, A. D., Rohaeti, E., \& } \\
\text { Prodjosantoso, A. K. (2018). } \\
\text { Promoting Critical Thinking } \\
\text { and Problem Solving Skills } \\
\text { of Preservice Elementary } \\
\text { Teachers through Process- } \\
\text { Oriented Guided-Inquiry } \\
\text { Learning (POGIL). } \\
\text { International Journal of } \\
\text { Instruction, 11(4). }\end{array}$ & $\begin{array}{l}\text { 1) Improve } \\
\text { students' higher } \\
\text { order thinking. } \\
\text { 2) Critical thinking } \\
\text { and problem } \\
\text { solving using } \\
\text { POGIL. }\end{array}$ & $\begin{array}{l}\text { POGIL } \\
\text { implementation: } \\
\text { Small groups with } \\
\text { roles assigned. } \\
\text { 2) To analyze, } \\
\text { explore and } \\
\text { establish a relation } \\
\text { between Critical } \\
\text { Thinking students } \\
\text { (CTS) and } \\
\text { Problem-solving } \\
\text { skills (PSS). }\end{array}$ & $\begin{array}{l}\text { 1) In-Class. } \\
\text { 2) Second-year } \\
\text { graduate students, } \\
\text { teaching Science } \\
\text { in Elementary } \\
\text { School Course. } \\
\text { 3) Two groups, } \\
\text { quasi- } \\
\text { experimental } \\
\text { group. } N=48 \\
\text { students. }\end{array}$ & $\begin{array}{l}\text { 1) Critical } \\
\text { Thinking Essay } \\
\text { Test (CTET) } \\
\text { 2) Problem- } \\
\text { Solving Essay } \\
\text { Test (PSET) } \\
\text { 3) Pretest- } \\
\text { posttest }\end{array}$ \\
\hline 3. & $\begin{array}{l}\text { Kim, S. S. (2018). m-POGIL } \\
\text { (modified-Process Oriented } \\
\text { Guided Inquiry Learning) } \\
\text { based Plastics Laboratory. }\end{array}$ & $\begin{array}{l}\text { 1) Improve } \\
\text { student learning \& } \\
\text { efficiency } \\
\text { 2) To explore real }\end{array}$ & $\begin{array}{l}\text { POGIL } \\
\text { implementation: } \\
\text { m-POGIL with } \\
\text { ABET criteria. }\end{array}$ & $\begin{array}{l}\text { 1) In-Class. } \\
\text { 2) Undergraduate } \\
\text { engineering } \\
\text { students. }\end{array}$ & Custom surveys \\
\hline
\end{tabular}




\begin{tabular}{|c|c|c|c|c|c|}
\hline & ARTICLE & THEME & $\begin{array}{c}\text { CRITICAL } \\
\text { COMPONENTS }\end{array}$ & CONTEXT & INSTRUMENTS \\
\hline & $\begin{array}{l}\text { Presented at the } 2018 \text { ASEE } \\
\text { Annual Conference \& } \\
\text { Exposition. }\end{array}$ & applications. & $\begin{array}{l}\text { Self \& Group. } \\
\text { Assigned roles. } \\
\text { 2) Lab } \\
\text { experiments to } \\
\text { discovery format } \\
\text { according to ATSM } \\
\text { and ISO standards. }\end{array}$ & $\begin{array}{l}\text { 3) } N=36 \\
\text { 4) Prelab and lab- } \\
\text { experiment }\end{array}$ & \\
\hline 4. & $\begin{array}{l}\text { Hu, H. H., Kussmaul, C., \& } \\
\text { Olivieri, L. M. (2018). } \\
\text { Special Session: Exploring } \\
\text { and Discovering Concepts } \\
\text { via POGIL. In Proceedings of } \\
\text { the 49th ACM Technical } \\
\text { Symposium on Computer } \\
\text { Science Education (pp. } \\
\text { 820-821). New York, NY, } \\
\text { USA: ACM. }\end{array}$ & $\begin{array}{l}\text { 1) Invent one's } \\
\text { own } \\
\text { understanding } \\
\text { 2) The instructor is } \\
\text { an active } \\
\text { facilitator. }\end{array}$ & $\begin{array}{l}\text { POGIL } \\
\text { implementation: } \\
\text { Small groups, } \\
\text { Teamwork, } \\
\text { Models } \\
\text { 2) Shifts attention } \\
\text { from language } \\
\text { syntax towards } \\
\text { conceptual } \\
\text { thinking. }\end{array}$ & $\begin{array}{l}\text { 1) In-Class } \\
\text { 2) } \\
\text { Faculty/Educators } \\
\text { 3) Students } \\
\text { involved in a } \\
\text { graduate course. }\end{array}$ & $\begin{array}{l}\text { No tools } \\
\text { mentioned. } \\
\text { \#ongoing project }\end{array}$ \\
\hline 5. & $\begin{array}{l}\text { Latimer, D. R., Ata, A., } \\
\text { Forfar, C. P., Kadhim, M., } \\
\text { Mcelrea, A., \& Sales, R. } \\
\text { (2018). Overcoming the } \\
\text { Hurdle from Undergraduate } \\
\text { Lab to Research Lab: A } \\
\text { Guided-Inquiry Structural } \\
\text { Characterization of a } \\
\text { Complex Mixture in the } \\
\text { Upper-Division } \\
\text { Undergraduate Organic } \\
\text { Lab. Journal of Chemical } \\
\text { Education,95(11), 2046- } \\
\text { 2049. }\end{array}$ & $\begin{array}{l}\text { 1) Help the } \\
\text { smooth transition } \\
\text { to a research lab. } \\
\text { 1) To build } \\
\text { deductive } \\
\text { reasoning skills } \\
\text { 2) Hybrid guided- } \\
\text { inquiry technique }\end{array}$ & $\begin{array}{l}\text { POGIL } \\
\text { implementation: } \\
\text { The "application" } \\
\text { phase of POGIL } \\
\text { similar } \\
\text { chromatographic } \\
\text { principles } \\
\text { Small groups }\end{array}$ & $\begin{array}{l}\text { 1) In-class } \\
\text { 2) Undergraduate } \\
\text { students. Third- } \\
\text { year organic } \\
\text { chemistry course. } \\
\text { 3) } N=34\end{array}$ & Unknown \\
\hline 6. & $\begin{array}{l}\text { Yuliastini, I. B., Rahayu, S., } \\
\text { Fajaroh, F., \& Mansour, N. } \\
\text { (2018). The effectiveness of } \\
\text { POGIL with SSI Context on } \\
\text { Vocational High School } \\
\text { Students' Chemistry } \\
\text { Learning } \\
\text { Motivation. Journal } \\
\text { Pendidikan IPA } \\
\text { Indonesia,7(1), 85-95. }\end{array}$ & $\begin{array}{l}\text { 1) Build learning } \\
\text { motivation } \\
\text { 2) Improve active } \\
\text { learning strategy. } \\
\text { 3) Ability to } \\
\text { solving contextual } \\
\text { problems }\end{array}$ & $\begin{array}{l}\text { POGIL } \\
\text { implementation } \\
\text { Small group work. } \\
\text { POGIL with Socio- } \\
\text { Scientific issues. }\end{array}$ & $\begin{array}{l}\text { 1) In-class } \\
\text { 2) Class } X \text { in the } \\
\text { State Vocational } \\
\text { High } \\
\text { 3) \#N value is not } \\
\text { given } \\
\text { 4) Quasi- } \\
\text { experimental } \\
\text { design with pre- } \\
\text { test - post-test } \\
\text { control group } \\
\text { design. }\end{array}$ & $\begin{array}{l}\text { Treatment } \\
\text { instruments: } \\
\text { Syllabus } \\
\text { Lesson Plans } \\
\text { Student } \\
\text { worksheet. } \\
\text { Measurement } \\
\text { instruments: } \\
\text { Learning } \\
\text { motivation } \\
\text { questionnaire. }\end{array}$ \\
\hline 8. & $\begin{array}{l}\text { Roller, M. C., Zori, S., \& } \\
\text { Lyons, E. (2018). The } \\
\text { Impact of Process-Oriented } \\
\text { Guided-Inquiry Learning } \\
\text { (POGIL) in Fundamental } \\
\text { and Medical Surgical } \\
\text { Nursing } 11 \text { Courses. }\end{array}$ & $\begin{array}{l}\text { 1) To develop } \\
\text { critical thinking } \\
\text { 2) To promote } \\
\text { Multidisciplinary } \\
\text { teamwork in } \\
\text { different fields. }\end{array}$ & $\begin{array}{l}\text { POGIL } \\
\text { implementation: } \\
\text { Small groups } \\
\text { Multi-disciplinary } \\
\text { teams }\end{array}$ & $\begin{array}{l}\text { 1) In-class } \\
\text { 2) Undergraduate } \\
\text { first-semester } \\
\text { nursing students } \\
\text { 3) } N=50 \\
\text { 4) Two groups, } \\
\text { purposive } \\
\text { sampling }\end{array}$ & $\begin{array}{l}\text { Comparative } \\
\text { GPAs }\end{array}$ \\
\hline 9 & $\begin{array}{l}\text { C Ezeala, C., A Ram, A., \& } \\
\text { Vulakouvaki, N. (2013). } \\
\text { Learning gain of pharmacy } \\
\text { students after introducing } \\
\text { guided inquiry learning with } \\
\text { computer simulation in a } \\
\text { pharmacology class in } \\
\text { Fiji. Journal of educational } \\
\text { evaluation for health }\end{array}$ & $\begin{array}{l}\text { 1) Determining } \\
\text { students learning } \\
\text { gain. } \\
\text { 2) Enhancing } \\
\text { analytical abilities. }\end{array}$ & $\begin{array}{l}\text { POGIL } \\
\text { implementation: } \\
\text { Group work: Roles } \\
\text { were assigned. } \\
\text { Supplementation } \\
\text { with simulation } \\
\text { practical sessions. } \\
\text { Tutor generated } \\
\text { practice problems }\end{array}$ & $\begin{array}{l}\text { 1) In-class } \\
\text { 2) } \mathrm{N}=42 \\
\text { 3) Second-year } \\
\text { Bachelor of } \\
\text { Pharmacy. } \\
\text { 4) pre and } \\
\text { posttests scores }\end{array}$ & Test Scores. \\
\hline
\end{tabular}




\begin{tabular}{|c|c|c|c|c|c|}
\hline & ARTICLE & THEME & $\begin{array}{c}\text { CRITICAL } \\
\text { COMPONENTS }\end{array}$ & CONTEXT & INSTRUMENTS \\
\hline & professions, 10, 9. & & & & \\
\hline $\begin{array}{l}1 \\
0\end{array}$ & $\begin{array}{l}\text { Villagonzalo, E. C. (2014). } \\
\text { Process oriented guided } \\
\text { inquiry learning: An } \\
\text { effective approach in } \\
\text { enhancing students' } \\
\text { academic performance. In } \\
\text { DLSU Research Congress } \\
\text { (Vol. 2, pp. 1-6). }\end{array}$ & $\begin{array}{l}\text { 1) Enhancing } \\
\text { academic } \\
\text { performance } \\
\text { 2) To develop } \\
\text { higher order } \\
\text { thinking skills. }\end{array}$ & $\begin{array}{l}\text { POGIL } \\
\text { implementation: } \\
\text { Small groups } \\
\text { POGIL materials } \\
\text { 2) Content and key } \\
\text { processes of } \\
\text { science. }\end{array}$ & $\begin{array}{l}\text { 1) In-class } \\
\text { 2) } N=41 \\
\text { 3) Two groups: } \\
\text { Pre-test posttest } \\
\text { randomized } \\
\text { control group. } \\
\text { 4)Third-year } \\
\text { special science } \\
\text { class. }\end{array}$ & $\begin{array}{l}\text { The Particulate } \\
\text { Nature of Matter } \\
\text { Assessment } \\
\text { version } 2 \\
\text { (ParNOMA2) }\end{array}$ \\
\hline $\begin{array}{l}1 \\
1\end{array}$ & $\begin{array}{l}\text { Douglas, E. P., \& C. C. } \\
\text { (2012). Process-oriented } \\
\text { Guided Inquiry Learning in } \\
\text { Engineering. Procedia - } \\
\text { Social and Behavioral } \\
\text { Sciences,56, 253-257. }\end{array}$ & $\begin{array}{l}\text { 1) Help to develop } \\
\text { the feeling of } \\
\text { ownership } \\
\text { 2)No lectures }\end{array}$ & $\begin{array}{l}\text { POGIL } \\
\text { Implementations: } \\
\text { Group work and } \\
\text { POGIL worksheets } \\
\text { containing. }\end{array}$ & $\begin{array}{l}\text { 1) In class } \\
\text { 2) } \mathrm{N}=217 \\
\text { 3) Undergraduate } \\
\text { class: Intro } \\
\text { Materials } \\
\text { Engineering } \\
\text { course. }\end{array}$ & $\begin{array}{l}\text { \#no tools were } \\
\text { mentioned. }\end{array}$ \\
\hline $\begin{array}{l}1 \\
2\end{array}$ & $\begin{array}{l}\text { Soltis, R., Verlinden, N., } \\
\text { Kruger, N., Carroll, A., \& } \\
\text { Trumbo, T. (2015). Process- } \\
\text { Oriented Guided Inquiry } \\
\text { Learning Strategy Enhances } \\
\text { Students' Higher Level } \\
\text { Thinking Skills in a } \\
\text { Pharmaceutical Sciences } \\
\text { Course. American Journal of } \\
\text { Pharmaceutical } \\
\text { Education,79(1), } 11 .\end{array}$ & $\begin{array}{l}\text { 1) Help build active } \\
\text { learning and } \\
\text { process skills } \\
\text { 3) Instructional } \\
\text { assessment. }\end{array}$ & $\begin{array}{l}\text { POGIL } \\
\text { Implementation: } \\
\text { Small group work } \\
\text { with roles } \\
\text { assigned. } \\
\text { 2) CAPE } 2013 \\
\text { outcomes }\end{array}$ & $\begin{array}{l}\text { 1) In-Class } \\
\text { 2) First-year } \\
\text { pharmacy } \\
\text { students } \\
\text { 3) } \mathrm{N}=112(2011) \text {, } \\
\mathrm{N}=111(2012) \\
\mathrm{N}=111(2013)\end{array}$ & $\begin{array}{l}\text { GPA was used } \\
\text { for evaluation. }\end{array}$ \\
\hline $\begin{array}{l}1 \\
3\end{array}$ & $\begin{array}{l}\text { Vanags, T., Pammer, K., \& } \\
\text { Brinker, J. (2013). Process- } \\
\text { oriented guided-inquiry } \\
\text { learning improves long- } \\
\text { term retention of } \\
\text { information. Advances in } \\
\text { Physiology Education,37(3), } \\
\text { 233-241. }\end{array}$ & $\begin{array}{l}\text { 1) Improve long } \\
\text { term retention of } \\
\text { concept } \\
\text { 2) Effects of new } \\
\text { facilitators. }\end{array}$ & $\begin{array}{l}\text { POGIL } \\
\text { Implementation: } \\
\text { Small group work } \\
\text { POGIL/NRO/NF } \\
\text { activity sheets } \\
\text { 2) new facilitator }\end{array}$ & $\begin{array}{l}\text { 1) In class } \\
\text { 2) Undergraduates } \\
\text { 3) } N=354 \\
\text { 4) Control and } \\
\text { experimental } \\
\text { (POGIL, NRO, NF) }\end{array}$ & $\begin{array}{l}\text { 1) Quasi- } \\
\text { experimental } \\
\text { design. } \\
\text { 2) Pretest and } \\
\text { posttest } \\
\text { assessment and } \\
\text { follow-up } \\
\text { quizzes }\end{array}$ \\
\hline $\begin{array}{l}1 \\
4 .\end{array}$ & $\begin{array}{l}\text { Brown, P. J. (2010). } \\
\text { Process-oriented guided- } \\
\text { inquiry learning in an } \\
\text { introductory anatomy and } \\
\text { physiology course with a } \\
\text { diverse student } \\
\text { population. Advances in } \\
\text { Physiology Education,34(3). }\end{array}$ & $\begin{array}{l}\text { 1) Help to build } \\
\text { confidence } \\
\text { 2) Working with } \\
\text { students of } \\
\text { diverse } \\
\text { background. }\end{array}$ & $\begin{array}{l}\text { POGIL } \\
\text { implementation: } \\
\text { Small groups with } \\
\text { assigned roles. } \\
\text { 2) Dealing with } \\
\text { Diverse } \\
\text { Backgrounds } \\
\end{array}$ & $\begin{array}{l}\text { 1) In-class } \\
\text { 2) Undergraduate } \\
\text { students } \\
\text { 3) } N=24 \text { (2008 } \\
\text { Spring), N = 18 } \\
\text { (2008 Fall), N = } 31 \\
\text { (2009 Spring), N = } \\
31 \text { (2009 Fall) }\end{array}$ & Custom survey. \\
\hline $\begin{array}{l}1 \\
5\end{array}$ & $\begin{array}{l}\text { Murray, T. A. (2013). } \\
\text { Teaching students to read } \\
\text { primary literature using } \\
\text { pogil } \\
\text { activities. Biochemistry and } \\
\text { Molecular Biology } \\
\text { Education,42(2), 165-173. } \\
\text { doi:10.1002/bmb.20765 }\end{array}$ & $\begin{array}{l}\text { 1) Help in building } \\
\text { confidence and } \\
\text { comfort. }\end{array}$ & $\begin{array}{l}\text { POGIL } \\
\text { implementation: } \\
\text { Pre-activity, in- } \\
\text { class and post } \\
\text { activity. } \\
\text { 2) Read and gain } \\
\text { experience, } \\
\text { recognize } \\
\text { connections and } \\
\text { differences } \\
\text { between results. }\end{array}$ & $\begin{array}{l}\text { 1) In-class } \\
\text { 2) } N=26 \text { (Fall } \\
\text { 2009) \& } \\
\quad N=13 \text { (Spring } \\
\text { 2010). } \\
\text { 3) pretest and } \\
\text { posttest scores }\end{array}$ & 1) SALG survey. \\
\hline $\begin{array}{l}1 \\
6\end{array}$ & $\begin{array}{l}\text { GALE, D. S., \& BOISSELLE, N. } \\
\text { L. (2015). The Effect of } \\
\text { POGIL on Academic } \\
\text { Performance and Academic } \\
\text { Confidence. Science }\end{array}$ & $\begin{array}{l}\text { 1) Help in building } \\
\text { academic } \\
\text { confidence. }\end{array}$ & $\begin{array}{l}\text { POGIL } \\
\text { implementation: } \\
\text { Small groups } \\
\text { (gender } \\
\text { differences) with }\end{array}$ & $\begin{array}{l}\text { 1) In-class } \\
\text { 2) Under graduate } \\
\text { students. } \\
\text { 3) } N=22 \\
\text { 4) Quasi- }\end{array}$ & Custom Survey \\
\hline
\end{tabular}




\begin{tabular}{|c|c|c|c|c|c|}
\hline & ARTICLE & THEME & $\begin{array}{c}\text { CRITICAL } \\
\text { COMPONENTS }\end{array}$ & CONTEXT & INSTRUMENTS \\
\hline & $\begin{array}{l}\text { Education } \\
\text { International,26(1), 56-61. }\end{array}$ & & $\begin{array}{l}\text { roles assigned. } \\
\text { 2) Ability to } \\
\text { conceptualize, } \\
\text { manipulate } \\
\text { concepts. }\end{array}$ & $\begin{array}{l}\text { experimental } \\
\text { design } \\
\text { 5) Pretest and } \\
\text { posttests scores }\end{array}$ & \\
\hline $\begin{array}{l}1 \\
7 .\end{array}$ & $\begin{array}{l}\text { SEN, Ş, \& YILMAZ, (2016). } \\
\text { The effect of Process } \\
\text { Oriented Guided Inquiry } \\
\text { Learning (POGIL) on 11th } \\
\text { Graders' conceptual } \\
\text { understanding of } \\
\text { electrochemistry. Asia- } \\
\text { Pacific Forum on Science } \\
\text { Learning and } \\
\text { Teaching,17(2). }\end{array}$ & $\begin{array}{l}\text { 1) Bolster } \\
\text { conceptual } \\
\text { change. } \\
\text { 2) Help in building } \\
\text { confidence level. }\end{array}$ & $\begin{array}{l}\text { POGIL } \\
\text { implementation: } \\
\text { Small groups with } \\
\text { roles assigned. } \\
\text { 2) Student- } \\
\text { Centered } \\
\text { approach. }\end{array}$ & $\begin{array}{l}\text { 1) In-class } \\
\text { 2) school students } \\
\text { 3) } N=115 \\
\text { 5) Experimental } \\
\text { and control group. }\end{array}$ & $\begin{array}{l}\text { 1) } \\
\text { Electrochemistry } \\
\text { concept test } \\
\text { (ECT) } \\
\text { Custom survey. }\end{array}$ \\
\hline $\begin{array}{l}1 \\
8\end{array}$ & $\begin{array}{l}\text { Trevathan, J., \& Myers, T. } \\
\text { (2013). Towards Online } \\
\text { Delivery of Process- } \\
\text { Oriented Guided Inquiry } \\
\text { Learning Techniques in } \\
\text { Information Technology } \\
\text { Courses. Journal of } \\
\text { Learning Design,6(2). }\end{array}$ & $\begin{array}{l}\text { 2) To observe } \\
\text { student } \\
\text { perceptions. }\end{array}$ & $\begin{array}{l}\text { POGIL } \\
\text { implementation: } \\
\text { Small groups with } \\
\text { roles assigned. } \\
\text { 1) Establish a } \\
\text { technological } \\
\text { platform } \\
\text { 2) Web } 2.0 \\
\text { technologies }\end{array}$ & $\begin{array}{l}\text { 1) Online class. } \\
\text { 2) Online third } \\
\text { year IT class }\end{array}$ & $\begin{array}{l}\text { Survey in the } \\
\text { form of: } \\
\text { Evaluations of } \\
\text { the Course (SEC) } \\
\text { Student } \\
\text { Evaluations of } \\
\text { the Teaching } \\
\text { SET) }\end{array}$ \\
\hline $\begin{array}{l}1 \\
9\end{array}$ & $\begin{array}{l}\text { CULTIVATING ICT } \\
\text { STUDENTS' } \\
\text { INTERPERSONAL SOFT } \\
\text { SKILLS IN ... (n.d.). } \\
\text { Retrieved from } \\
\text { https://files.eric.ed.gov/full } \\
\text { text/EJ1048767.pdf }\end{array}$ & $\begin{array}{l}\text { 1) Develop } \\
\text { team building } \\
\text { 2) Improve } \\
\text { interpersonal skills }\end{array}$ & $\begin{array}{l}\text { POGIL } \\
\text { implementation } \\
\text { Small groups with } \\
\text { roles assigned. } \\
\text { Blogs and social } \\
\text { media } \\
\text { Group work and } \\
\text { informal peer } \\
\text { reviewed } \\
\text { Group work }\end{array}$ & $\begin{array}{l}\text { 1) Online } \\
\text { 2)synchronous } \\
\text { (Blackboards } \\
\text { Collaborate) and } \\
\text { asynchronous } \\
\text { online } \\
\text { technologies } \\
\text { (Blogs and Media) } \\
\text { 3) } N=300\end{array}$ & $\begin{array}{l}\text { \#No Tools were } \\
\text { mentioned. }\end{array}$ \\
\hline $\begin{array}{l}2 \\
1\end{array}$ & $\begin{array}{l}\text { Process-Oriented Guided- } \\
\text { Inquiry Learning In Financial } \\
\text {... (n.d.). Retrieved from } \\
\text { https://www.theforumjour } \\
\text { nal.org/wp- } \\
\text { content/uploads/2018/05/ } \\
\text { Process-Oriented.pdf }\end{array}$ & $\begin{array}{l}\text { 1) ) Enhancing } \\
\text { academic } \\
\text { performance }\end{array}$ & $\begin{array}{l}\text { POGIL } \\
\text { implementation } \\
\text { Four groups with } \\
\text { assigned roles } \\
5 \text { assignments } \\
\text { were handed out } \\
\text { to both groups }\end{array}$ & $\begin{array}{l}\text { 1) Online } \\
\text { 2) Undergraduate } \\
\text { class. } \\
\text { 3) } N=102 \\
\text { 3) Comparative } \\
\text { study between } \\
\text { hybrid and lecture } \\
\text { group. }\end{array}$ & $\begin{array}{l}\text { Assignemt } \\
\text { scores were } \\
\text { used for analysis. }\end{array}$ \\
\hline $\begin{array}{l}2 \\
2\end{array}$ & $\begin{array}{l}\text { Cold, S. J. (2013). Partially } \\
\text { flipped. Proceedings of the } \\
\text { 13th Annual ACM SIGITE } \\
\text { Conference on Information } \\
\text { Technology Education - } \\
\text { SIGITE 13. } \\
\text { doi:10.1145/2512276.2512 } \\
314\end{array}$ & $\begin{array}{l}\text { 1) To imbibe } \\
\text { peripheral } \\
\text { learning. } \\
\text { 2) To build process } \\
\text { skills. }\end{array}$ & $\begin{array}{l}\text { POGIL } \\
\text { implementation } \\
\text { Small groups with } \\
\text { roles assigned }\end{array}$ & $\begin{array}{l}\text { 1) Online } \\
\text { 2) Flipped } \\
\text { undergraduate } \\
\text { class. }\end{array}$ & $\begin{array}{l}\text { \#no tools were } \\
\text { mentioned }\end{array}$ \\
\hline
\end{tabular}


Most articles in the last decade on POGIL implementation focused on three main themes - critical and other higher-order thinking, problem-solving skills, and building student confidence. Most papers used well-known, standardized instruments to measure the impact of POGIL implementations. A few papers developed custom surveys, but even those usually had theoretical underpinnings. A few studies with experimental design relied on pre-test/post-test evaluations to measure the change due to POGIL intervention. Only one paper out of the 18 which we reviewed was in an online setting (Trevathan \& Myers, 2013), making our contribution to POGIL in the online setting unique and among the early ideas in online implementation. We will discuss the critical components in section 5, where we describe Cyber POGIL and some of the tweaks required to the critical components for the online setting.

\section{POGIL implementation context and methodology}

At IUPUI's HIM program, we have taken multiple approaches such as - using active learning, problem-based learning, experiential learning and inquiry learning, to involve the undergraduate students in the learning process. These pedagogical methods try to move away from didactic content delivery towards engaging students in their own learning process., We implemented POGIL in two HIM courses, M200 Database design and M220- Health Informatics for Decision Support. POGIL practices were integrated into the curriculum redesign of these HIM courses. We implemented the core philosophy of POGIL in the two courses - students learn through the process of performing activities that aid in developing critical thinking skills, as in such scenarios, learning is by doing, and the teacher does not instruct, he/she facilitates guided learning. Before modifying the courses, active learning strategies such as group discussion on database-related work, and virtual labs, where students critically review decision support tools were already part of the curriculum. Based on the HIM plan of study, these courses are taken by students in the 2nd year of their BS in HIM. The HIM M200 is a general education course, which can be taken by students from any undergraduate program at IUPUI. Approximately 40 students enroll in these courses each semester.

We presently use OpenMRS, an open-source EHR system in the undergraduate HIM and the graduate Health Informatics courses at IUPUI. OpenMRS is a popular EHR system used in over 42 countries, primarily designed for low- and middle-income countries, but is also used in some academic health centers and schools of informatics in high-income countries. The two HIM courses provide didactic content on the Canvas learning management system (LMS) in the form of lecture slides and video recordings. This content gives background information required to synthesize the new concept that we expect the students to develop and understand using the POGIL pedagogy. The POGIL implementation began by dividing the class into groups of 3 to 4 students. Each student of a group can play two roles - iTrainee and rTrainee. The "inquiring student" called the iTrainee is asked to create a set of tasks based on the concept that will be discovered based on the background information provided in the lecture material and instructions from the instructor. The iTrainee is not aware of the most efficient way to perform the task but tries to perform the task on their own, based on the concepts explained in the lecture slides. See Figure 1 below for example. After performing the task, the iTrainee requests the other students of their group (rTrainees) to perform the same task. In creating this request, the iTrainee does not describe how the task was performed. Instead, the iTrainee defines a question that will be answered by performing the task.
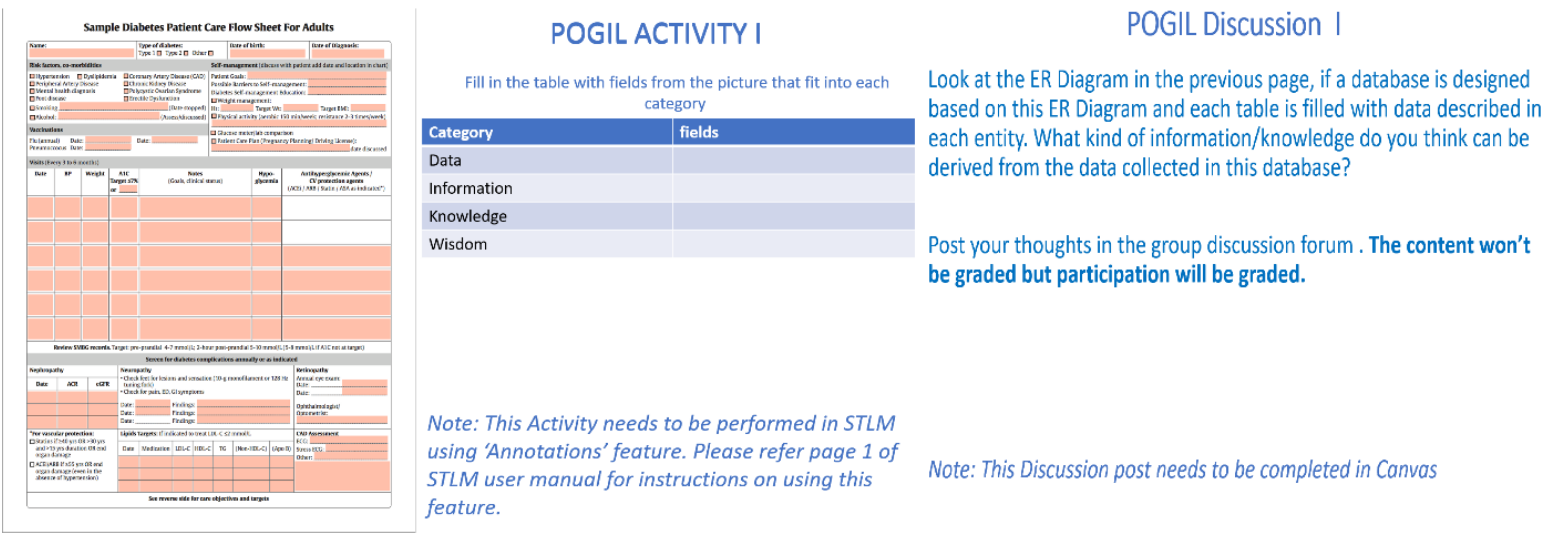

Note: This Activity needs to be performed in STLM

using 'Annotations' feature. Please refer page 1 of Note: This Discussion post needs to be completed in Canvas
STLM user manual for instructions on using this feature.

Figure 1: A comparison of slide material (right), STLM (left), and the Canvas discussion

Reference this paper: Purkayastha, S. et al., 2019. Critical Components of Formative Assessment in Process-Oriented Guided Inquiry Learning for Online Labs. The Electronic Journal of e-Learning, 17(2), pp. 79-92, available online at www.ejel.org 
Let us look at the example POGIL activity from Figure 1. The HIM M220 course has an assignment, which requires all the students to identify data/metadata, information, and knowledge from the EHR data shown in the patient dashboard, such that they can be used to create clinical decision support rules. We modified the assignment in such a way, where iTrainee is given a set of instructions to search for a patient in the EHR system and annotate the parts of the patient record values as data or information or knowledge. The STLM tool enables selecting or typing text in the EHR forms and tag them as data/metadata, information or knowledge. The task that was performed by the iTrainee is then posted on the Canvas LMS. The rest of the group members are notified of this request. Other "responding students" called rTrainee now attempt to complete the task that is put forth by the question of the iTrainee. The rTrainees attempt to complete the task, without knowing how the iTrainee completed the task. After completion of the given task, the rTrainees and iTrainee will be able to compare their work with other members of the group. The iTrainee will also similarly have to play the role of a rTrainee when other members of his/her group make inquiries and propose new tasks to the group. We found that with each attempt as a rTrainee, there is improved student learning of the concept, followed by knowledge reconstruction that occurs by observing the comparison of the tasks performed by different students.

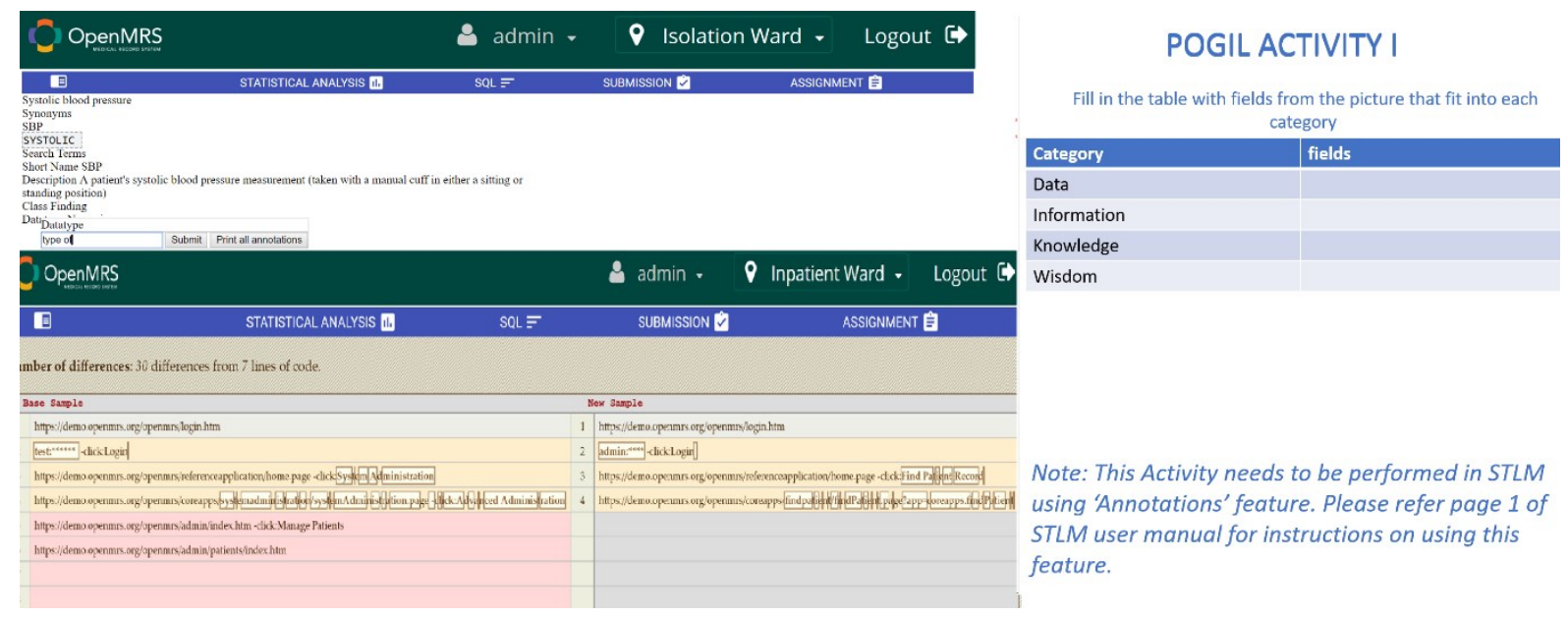

Figure 2: Screenshot showing STLM tool with annotation and compare feature (left) and POGIL task (right)

Figure 2. shows how this task is performed by the student in the EHR and monitored by the STLM

An example POGIL task: Let us look at the step-by-step process by which an iTrainee needs to reach a patient record and label parts of the patient record, such as to identify data, information, or knowledge. In this POGIL activity, the iTrainee when performing this task has to gain a process skill through which they gain three planned concepts that are relevant for the basics of clinical decision support systems.

1. The process skills of finding a patient record helps articulate the concept of identifiers through which a patient can be searched and identified. In the didactic content of the lecture, the class has been shown a tutorial describing the UI of the OpenMRS EHR system. However, the concept of patient identifiers is new, and something the students must discover on their own. The iTrainee might search by name, some medical record number (MRN), etc. and reach the patient record. This search term, if not unique, will result in the rTrainees finding different patients when the rTrainee gives the task. Thus, the iTrainee has to be careful in articulating that patient identifiers work best when they can uniquely identify patients. Therefore, after the iTrainee has looked up for a patient by their MRN (say 100-8), the task that they define for example: "Please list the date of birth, BMI and condition based on the patient vitals of patient 100-8."

2. The process skill of opening the vitals section of a patient record helps articulate the concept of forms in the OpenMRS EHR. The iTrainee needs to understand that data can be captured through multiple forms, and one of the first forms in the EHR workflow is the vitals form, which captures height, weight, blood pressure, etc. The iTrainee also distinguishes that demographics such as name, age, etc. are semi-permanent data, which will not be captured very often using forms. Thus, when they define the question: "Please list the date of birth, BMI and condition based on the patient vitals of patient 1008 ", they are formulating the forms concept in their knowledge construction.

3. The process skill of looking through the vitals data and identifying data, information, and knowledge is the next step in this POGIL activity. The iTrainee has learned from the didactic content of the lecture 
that data is unprocessed such as recording date of birth of the patient. When data is processed it becomes information, such as when height and weight are combined in a formula to calculate BMI. And when you draw inferences from the information, it becomes knowledge. For example, when BMI is higher than 25 , the condition of the patient is overweight. Thus, the question - "Please list the date of birth, BMI and condition based on the patient vitals of patient $100-8$ ".

The iTrainee may or may not have understood these concepts, and might create a half-baked or incomplete question. Still, the iTrainee posts the question on the Canvas LMS discussion forum for other members of the group, who will now have to play the role of rTrainees and perform the same task and gain the process skills. The iTrainee instructs the rTrainees with the specific patient MRN to search, the form that needs to be opened and the type of concept or data needed to complete the task. After completion of the task, students can compare their methods to attempt the task with other members of the group. Instead of the instructor providing formative assessment here, the rTrainees might see that concepts identified by the iTrainee might not be complete. Thus, they discuss how each of the above mentioned three concepts can be different, and the question and the task can all be performed differently by them compared to the iTrainee's original task. This monitoring and comparison is facilitated by the STLM tool. The POGIL implementation involves students in the learning process, through inquiry and activities of the constructivist learning cycle. Due to the use of the POGIL approach, students discover different ways to complete the tasks in the EHR system. This is difficult in a didactic setting since all possible ways to solve the problem are hard to demonstrate. Whereas in a POGIL approach environment, the students will work with/against (in a competitive way) to solve the problem and discover efficient ways to complete a task. The STLM tool provides many features that are appropriate for the two courses (Purkayastha, Surapaneni, et al., 2018), but it is currently being expanded for use in training clinicians in discovering appropriate ways to use EHR systems, and learning by observing and comparing with other users of the EHR (Purkayastha, Naliyatthaliyazchayil, Surapaneni, Kowkutla, \& Maity, 2018).

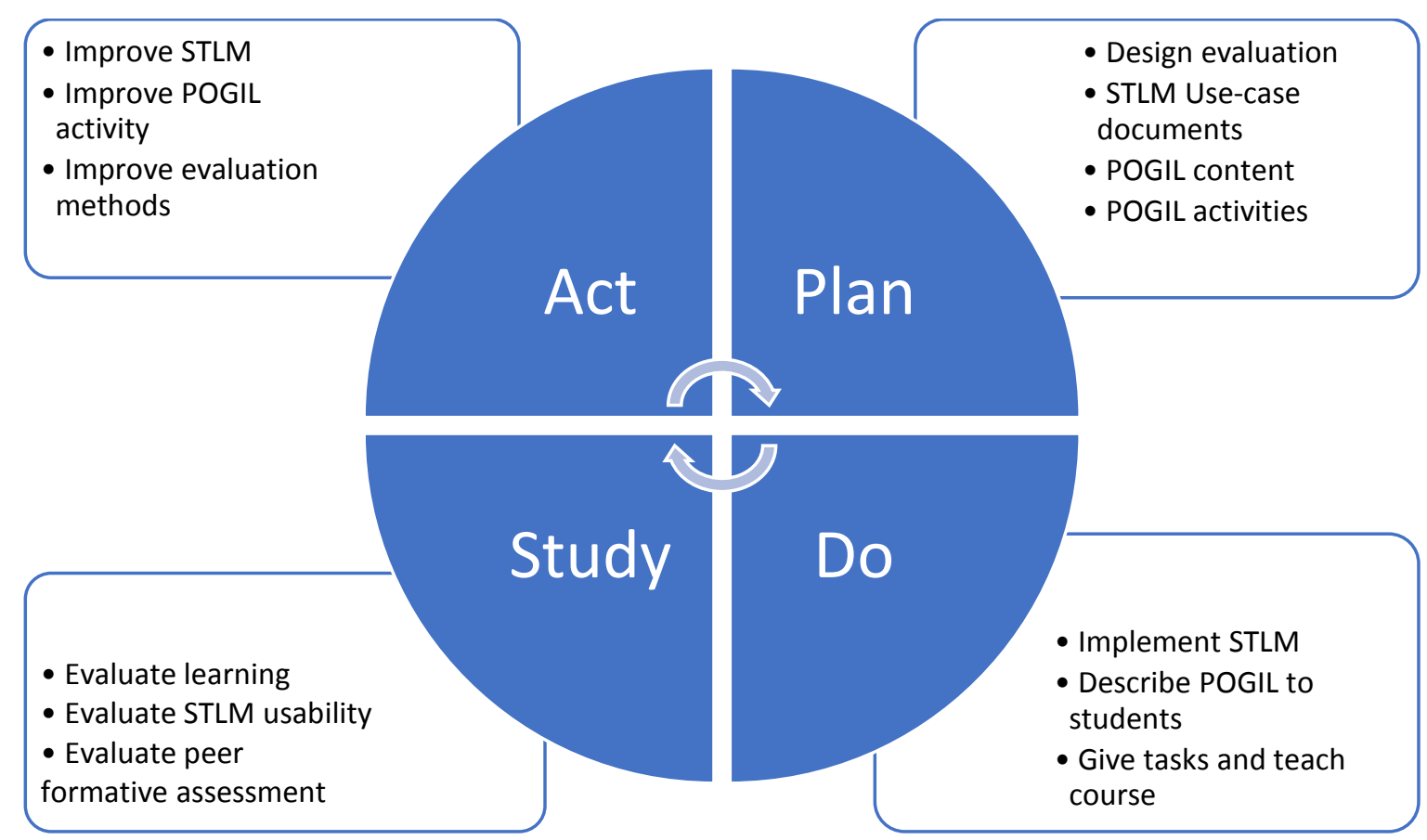

Figure 3: The Plan-Do-Study-Act cycle for POGIL implementation methodology in the courses

The POGIL pedagogy in the two courses has been in the implementation of three iterations of Plan-Do-StudyAct (PDSA) from Fall 2017 to Spring 2019. The PDSA or as it is sometimes also referred to as Plan-Do-CheckAdjust is an iterative four-step management method for continuous improvement. It is also called the Deming circle/cycle (Moen \& Norman, 2006) or the Shewhart cycle (Best \& Neuhauser, 2006). As part of the Plan phase, we performed initially a 4-5 month brainstorming period with a 5-member team consisting of two instructors, one education researcher, and two graduate students who were developers of the system. The lecture slides and videos were updated with POGIL activities and updates to the didactic content, by removing the introduction of new concepts, and replacing them by background information that was required to do the 
POGIL activities. The use-case documents listing the STLM features were created and improved in every iteration of the Plan phase. The STLM updates were deployed on OpenMRS in the Do phase, along with describing to the students about POGIL and the tasks and roles that they will play as part of the course pedagogy. Finally, the Do phase has the teaching of the course and the quizzes, forum discussions, and assignments that are part of the course assessments. The Study phase consists of evaluating student learning gains through a comparison of grades in the course assessments before and after the implementation of POGIL. We have a 10 question survey, along with a volunteer interview with the students to improve the STLM tool, and how they found the features of the tool. We also evaluated the formative peer assessment that is part of the discussion forums and comparison of the POGIL activity tasks using the STLM tool, and the number of cycles required for the student groups to grasp the planned concepts. The instructor and the researchers studied how the iTrainee and rTrainees designed and improved the tasks within a couple of weeks when they were given the task, and the students ask the question along with the process to complete the task. The Act phase is used to improve the STLM features based on the feedback, change POGIL activities that required a lot of cycles to solidify the planned concepts, as well as improve our evaluation surveys, and interview guides. By performing the PDSA cycles, we can articulate some of the critical components that are necessary to implement POGIL in an online setting, where hands-on and practical lab sessions are a bulk of the course duration.

\section{Critical components of Cyber POGIL}

From the summary that we presented in Table 1 and our experience, we see that many critical components of POGIL can be implemented in the online setting. However, certain parts require tweaking, particularly those that involve the instructor role as a facilitator and student role in group-based learning.

Larger group sizes: Many papers from Table 1 identify small group size as a critical component for the implementation of POGIL. Many researchers used small groups (Hu, Kussmaul, \& Olivieri, 2018; Smith, Paddock, Vaughan, \& Parkin, 2018; Vanags, Pammer, \& Brinker, 2013; Villagonzalo, 2014; Yuliastini, Rahayu, Fajaroh, \& Mansour, 2018), as a way to build confidence among students that they can construct their own knowledge. While small groups are an essential part of POGIL pedagogy, there are other researchers who use it to either create multi-disciplinary teams (Brown, 2010; Roller, Zori, \& Lyons, 2018) or assign specific roles (C Ezeala, A Ram, \& Vulakouvaki, 2013; Kim, 2018; Saputro, Rohaeti, \& Prodjosantoso, 2018; Soltis, Verlinden, Kruger, Carroll, \& Trumbo, 2015). In our experience of implementing POGIL in the online setting, we observed that there was very little difference in group behavior, measured through the content of forum posts or the learning gains between 3-member teams and 10-member teams. If anything, with smaller teams, there were more iterations captured in the STLM tool that were required to solidify the planned concepts, compared to in larger teams. This might be peculiar to the classes or the subject area, but still, the online setting allows for much larger groups of students to communicate asynchronously, even though there are more follow-up threads and replies. We do not suggest that MOOCs can be organized in this fashion but hypothesize that at least group sizes of 10 students could be accommodated in Cyber POGIL implementations.

Limited active tutoring: Not completely following the POGIL principle that the teacher is only a facilitator in the classroom, quite often POGIL implementations introduce concepts through didactic material and then solidify those through groups through process skills (C Ezeala et al., 2013; Latimer et al., 2018; Villagonzalo, 2014). This is mainly seen in chemistry lab sessions, where a tutor supports the student groups. In Cyber POGIL, we have not found as much use of active tutoring, both from the standpoint of time-space differences between the students, as well as the POGIL activity. Cyber POGIL requires a much thorough tutorial that will demonstrate how an activity might be performed in the virtual lab since active tutoring cannot be provided.

Independent process skills: POGIL focuses on group construction of knowledge, and the use of peers from the groups as a way to correct and solidify the understanding of concepts. Still, researchers have used tutors, senior peers, or instructors to provide process skills. Cyber POGIL allows for student development of more independent process skills. This is likely due to the problem-solving nature of the POGIL activities in our courses, and we observed that there were multiple ways in which students were able to solve the same problems. While other physical sciences like in Chemistry or pharmacology might not have many different solutions to a given problem, programming tasks might have many possible solutions. Thus, Cyber POGIL lends itself for IT or programming-related courses, and the students gain the process skills more independently than the other POGIL implementations. 
Critical role of monitoring tool: Unlike physical, in-class implementations of POGIL where equipments (in Chemistry) or manikins (in Nursing) do not play a significant role, Cyber POGIL critically depend on the monitoring tool through which students will perform the activities. This demonstrates a dual nature of the technology - as both a supportive tool, as well as a dependency for the learning. Unless the tools are custommade for the content of the course, students will get stuck with the usability problems with the tool, instead of focusing on learning the concepts from the course.

\section{Conclusion}

Other schemes used for engagement and socialization of the online students are Flipped Classroom, Active Learning and Communicative language teaching. The flipped classroom settings promotes student centered learning approach and collaboration, but it also can create or lead to a digital divide. The success of this scheme relies on preparation and trust. Active learning occur in many forms, such as Discovery Learning, project based learning and cooperative learning are a few techniques that fall under this schemes. This learning form is goal driven and activity based learning. However it has been observed that these activities are time taking. Communicative language teaching is an approach to language teaching that focalizes on the concept of interaction as both means and ultimate goal of study.

Cyber POGIL might be a requirement for the evolution of the guided inquiry learning pedagogy in the future. The critical components that are different for Cyber POGIL such as large student groups, limited active tutoring, independent process skills and the role of the monitoring tool are different from traditional in-class POGIL implementations. The usual critical components of the teacher as a facilitator, specific POGIL activities that teach process skills, critical thinking and team-based learning strategies need to be implemented in Cyber POGIL too. We have implemented the Cyber POGIL in two courses only, that is not enough to identify all critical components of cyber POGIL. This is considered as a limitation of our study. We hope many other researchers, including the POGIL project itself, can produce more evidence about the implementation of POGIL in online settings.

\section{References}

Anderson, T., \& Dron, J. (2011). Three generations of distance education pedagogy. The International Review of Research in Open and Distributed Learning, 12(3), 80-97. https://doi.org/10.19173/irrodl.v12i3.890

Baleni, Z. G. (2015). Online Formative Assessment in Higher Education: Its Pros and Cons. Electronic Journal of E-Learning, $13(4), 228-236$.

Bell, B., \& Cowie, B. (2001). The characteristics of formative assessment in science education. Science Education, 85(5), 536-553. https://doi.org/10.1002/sce.1022

Bennett, R. E. (2011). Formative assessment: a critical review. Assessment in Education: Principles, Policy \& Practice, 18(1), 5-25. https://doi.org/10.1080/0969594X.2010.513678

Best, M., \& Neuhauser, D. (2006). Walter A Shewhart, 1924, and the Hawthorne factory. BMJ Quality \& Safety, 15(2), 142143. https://doi.org/10.1136/qshc.2006.018093

Black, P., \& Wiliam, D. (2009). Developing the theory of formative assessment. Educational Assessment, Evaluation and Accountability(Formerly: Journal of Personnel Evaluation in Education), 21(1), 5. https://doi.org/10.1007/s11092-0089068-5

Bloomrosen, M., \& Berner, E. S. (2017). Findings from the 2017 Yearbook Section on Health Information Management. Yearbook of Medical Informatics, 26(01), 78-82.

Briggs, D. C., Ruiz-Primo, M. A., Furtak, E., Shepard, L., \& Yin, Y. (2012). Meta-Analytic Methodology and Inferences About the Efficacy of Formative Assessment. Educational Measurement: Issues and Practice, 31(4), 13-17. https://doi.org/10.1111/j.1745-3992.2012.00251.x

Brown, P. J. (2010). Process-oriented guided-inquiry learning in an introductory anatomy and physiology course with a diverse student population. Advances in Physiology Education, 34(3), 150-155.

C Ezeala, C., A Ram, A., \& Vulakouvaki, N. (2013). Learning gain of pharmacy students after introducing guided inquiry learning with computer simulation in a pharmacology class in Fiji. Journal of Educational Evaluation for Health Professions, 10. https://doi.org/10.3352/jeehp.2013.10.9

Chattopadhyay, S., Shankar, S., Gangadhar, R. B., \& Kasinathan, K. (2018). Applications of Artificial Intelligence in Assessment for Learning in Schools. Handbook of Research on Digital Content, Mobile Learning, and Technology Integration Models in Teacher Education, 185-206. https://doi.org/10.4018/978-1-5225-2953-8.ch010

Cowie, B., \& Bell, B. (1999). A Model of Formative Assessment in Science Education. Assessment in Education: Principles, Policy \& Practice, 6(1), 101-116. https://doi.org/10.1080/09695949993026

Delquadri, J., Greenwood, C. R., Whorton, D., Carta, J. J., \& Hall, R. V. (1986). Classwide Peer Tutoring. Exceptional Children, 52(6), 535-542. https://doi.org/10.1177/001440298605200606 
Dixson, M. D., Greenwell, M. R., Rogers-Stacy, C., Weister, T., \& Lauer, S. (2017). Nonverbal immediacy behaviors and online student engagement: bringing past instructional research into the present virtual classroom. Communication Education, 66(1), 37-53. https://doi.org/10.1080/03634523.2016.1209222

Dunn, K. E., \& Mulvenon, S. W. (2009). A critical review of research on formative assessment: The limited scientific evidence of the impact of formative assessment in education. Practical Assessment, Research \& Evaluation, 14(7), 111.

Dutton, P. J., Bickerstaff, H. E., Rymer, J. M., Webb, M. E., Ballinger-Mills, D., Greenough, A., \& Reynolds, P. A. (2017). Investigation of Formative Assessment of Learning (INFORMAL): The Performance Indicator Tool (PIT). Technology, Knowledge and Learning, 22(2), 161-171. https://doi.org/10.1007/s10758-017-9307-2

Friðriksdóttir, K. (2018). The impact of different modalities on student retention and overall engagement patterns in open online courses. Computer Assisted Language Learning, 31(1-2), 53-71. https://doi.org/10.1080/09588221.2017.1381129

Gibson, C. J., Dixon, B. E., \& Abrams, K. (2015). Convergent evolution of health information management and health informatics. Applied Clinical Informatics, 06(1), 163-184. https://doi.org/10.4338/ACl-2014-09-RA-0077

Gikandi, J. W., Morrow, D., \& Davis, N. E. (2011). Online formative assessment in higher education: A review of the literature. Computers \& Education, 57(4), 2333-2351. https://doi.org/10.1016/j.compedu.2011.06.004

Graesser, A., \& McDaniel, B. (2017). Conversational agents can provide formative assessment, constructive learning, and adaptive instruction. In The Future of Assessment (pp. 85-112). Routledge.

Green, N. C., Edwards, H., Wolodko, B., Stewart, C., Brooks, M., \& Littledyke, R. (2010). Reconceptualising higher education pedagogy in online learning. Distance Education, 31(3), 257-273. https://doi.org/10.1080/01587919.2010.513951

Hamodi, C., López-Pastor, V. M., \& López-Pastor, A. T. (2017). If I experience formative assessment whilst studying at university, will I put it into practice later as a teacher? Formative and shared assessment in Initial Teacher Education (ITE). European Journal of Teacher Education, 40(2), 171-190. https://doi.org/10.1080/02619768.2017.1281909

Hanson, D. M. (2006). Instructor's guide to process-oriented guided-inquiry learning. Citeseer.

Hooshyar, D., Ahmad, R. B., Yousefi, M., Fathi, M., Horng, S.-J., \& Lim, H. (2016). Applying an online game-based formative assessment in a flowchart-based intelligent tutoring system for improving problem-solving skills. Computers \& Education, 94, 18-36. https://doi.org/10.1016/j.compedu.2015.10.013

Hu, H. H., Kussmaul, C., \& Olivieri, L. M. (2018). Special Session: Exploring and Discovering Concepts via POGIL. In Proceedings of the 49th ACM Technical Symposium on Computer Science Education (pp. 820-821). New York, NY, USA: ACM. https://doi.org/10.1145/3159450.3159637

Janke, T., \& Varma-Nelson, P. (2014). Cyber Peer-Led Team Learning (cPLTL). In EdMedia: World Conference on Educational Media and Technology (pp. 29-34). Association for the Advancement of Computing in Education (AACE).

Kim, S. S. (2018). m-POGIL (modified-Process Oriented Guided Inquiry Learning) based Plastics Laboratory. Presented at the 2018 ASEE Annual Conference \& Exposition. Retrieved from https://peer.asee.org/m-pogil-modified-process-orientedguided-inquiry-learning-based-plastics-laboratory

Latimer, D. R., Ata, A., Forfar, C. P., Kadhim, M., McElrea, A., \& Sales, R. (2018). Overcoming the Hurdle from Undergraduate Lab to Research Lab: A Guided-Inquiry Structural Characterization of a Complex Mixture in the UpperDivision Undergraduate Organic Lab. Journal of Chemical Education, 95(11), 2046-2049. https://doi.org/10.1021/acs.jchemed.7b00421

Lau, A. M. S. (2016). 'Formative good, summative bad?' - A review of the dichotomy in assessment literature. Journal of Further and Higher Education, 40(4), 509-525. https://doi.org/10.1080/0309877X.2014.984600

Lull, M. E., \& Mathews, J. L. (2016). Online Self-testing Resources Prepared by Peer Tutors as a Formative Assessment Tool in Pharmacology Courses. American Journal of Pharmaceutical Education, 80(7), 124. https://doi.org/10.5688/ajpe807124

Mauser, K., Sours, J., Banks, J., Newbrough, J. R., Janke, T., Shuck, L., ... Varma-Nelson, P. (2011). Cyber peer-led team learning (CPLTL): Development and implementation. EDUCAUSE Review Online, 34(4), 1-17.

McFarland, J., Hussar, B., Wang, X., Zhang, J., Wang, K., Rathbun, A., ... Mann, F. B. (2018). The Condition of Education 2018. NCES 2018-144. National Center for Education Statistics. Retrieved from https://eric.ed.gov/?id=ED583502

McLaughlin, T., \& Yan, Z. (2017). Diverse delivery methods and strong psychological benefits: A review of online formative assessment. Journal of Computer Assisted Learning, 33(6), 562-574. https://doi.org/10.1111/jcal.12200

Mingfei, L., \& Jie, Z. (2010). Study on the Mechanisms of Team Learning upon Knowledge Transfer: A Research Based on Social Constructivism Learning Theory. In 2010 3rd International Conference on Information Management, Innovation Management and Industrial Engineering (Vol. 1, pp. 196-200). https://doi.org/10.1109/ICIII.2010.53

Moen, R., \& Norman, C. (2006). Evolution of the PDCA cycle. Citeseer.

Moog, R. S., Spencer, J. N., \& Straumanis, A. R. (2006). Process-Oriented Guided Inquiry Learning: POGIL and the POGIL Project. Metropolitan Universities, 17(4), 41-52.

Nicol, D. J., \& Macfarlane-Dick, D. (2006). Formative assessment and self-regulated learning: a model and seven principles of good feedback practice. Studies in Higher Education, 31(2), 199-218. https://doi.org/10.1080/03075070600572090

Offerdahl, E. G., McConnell, M., \& Boyer, J. (2018). Can I Have Your Recipe? Using a Fidelity of Implementation (FOI) Framework to Identify the Key Ingredients of Formative Assessment for Learning. CBE-Life Sciences Education, 17(4), es16. https://doi.org/10.1187/cbe.18-02-0029

Parmelee, D., Michaelsen, L. K., Cook, S., \& Hudes, P. D. (2012). Team-based learning: A practical guide: AMEE Guide No. 65. Medical Teacher, 34(5), e275-e287. https://doi.org/10.3109/0142159X.2012.651179 
Pereira, D., Flores, M. A., \& Niklasson, L. (2016). Assessment revisited: a review of research in Assessment and Evaluation in Higher Education. Assessment \& Evaluation in Higher Education, 41(7), 1008-1032. https://doi.org/10.1080/02602938.2015.1055233

Perkins, D. V., \& Saris, R. N. (2001). A “Jigsaw Classroom” Technique for Undergraduate Statistics Courses. Teaching of Psychology, 28(2), 111-113. https://doi.org/10.1207/S15328023TOP2802_09

Purkayastha, S., Naliyatthaliyazchayil, P. R. M., Surapaneni, A. K., Kowkutla, A., \& Maity, P. (2018). Improving “Desktop medicine" efficiency using Guided Inquiry Learning in an Electronic Health Records System.

Purkayastha, S., Surapaneni, A. K., \& Maity, P. (2018). Implementing Guided Inquiry Learning and Measuring Engagement Using an Electronic Health Record System in an Online Setting. In European Conference on e-Learning; Kidmore End (pp. 481-488). Kidmore End, United Kingdom, Kidmore End: Academic Conferences International Limited. Retrieved from https://search.proquest.com/docview/2154982629/abstract/1FB824CA3CDF4580PQ/1

Roller, M. C., Zori, S., \& Lyons, E. (2018). The Impact of Process-Oriented Guided-Inquiry Learning (POGIL) in Fundamental and Medical Surgical Nursing 11 Courses. Retrieved from https://sigma.nursingrepository.org/handle/10755/623952

Sadler, D. R. (1989). Formative assessment and the design of instructional systems. Instructional Science, 18(2), $119-144$. https://doi.org/10.1007/BF00117714

Sadler, D. R. (1998). Formative Assessment: revisiting the territory. Assessment in Education: Principles, Policy \& Practice, 5(1), 77-84. https://doi.org/10.1080/0969595980050104

Santamaría Lancho, M., Hernández, M., Sánchez-Elvira Paniagua, Á., Luzón Encabo, J. M., \& de Jorge-Botana, G. (2018). Using Semantic Technologies for Formative Assessment and Scoring in Large Courses and MOOCs. Journal of Interactive Media in Education, 2018(1). Retrieved from https://eric.ed.gov/?id=EJ1190185

Saputro, A. D., Rohaeti, E., \& Prodjosantoso, A. K. (2018). Promoting Critical Thinking and Problem Solving Skills of Preservice Elementary Teachers through Process-Oriented Guided-Inquiry Learning (POGIL). International Journal of Instruction, 11(4).

Smith, A. L., Paddock, J. R., Vaughan, J. M., \& Parkin, D. W. (2018). Promoting Nursing Students' Chemistry Success in a Collegiate Active Learning Environment: "If I Have Hope, I Will Try Harder." Journal of Chemical Education, 95(11), 1929-1938. https://doi.org/10.1021/acs.jchemed.8b00201

Soltis, R., Verlinden, N., Kruger, N., Carroll, A., \& Trumbo, T. (2015). Process-Oriented Guided Inquiry Learning Strategy Enhances Students' Higher Level Thinking Skills in a Pharmaceutical Sciences Course. American Journal of Pharmaceutical Education, 79(1), 11. https://doi.org/10.5688/ajpe79111

Spector, J. M., Ifenthaler, D., Sampson, D., Yang, L. (Joy), Mukama, E., Warusavitarana, A., ... Gibson, D. C. (2016). Technology Enhanced Formative Assessment for 21st Century Learning. Journal of Educational Technology \& Society, 19(3), 58-71.

Stains, M., \& Vickrey, T. (2017). Fidelity of Implementation: An Overlooked Yet Critical Construct to Establish Effectiveness of Evidence-Based Instructional Practices. CBE Life Sciences Education, 16(1). https://doi.org/10.1187/cbe.16-03-0113

Trevathan, J., \& Myers, T. (2013). Towards online delivery of process-oriented guided inquiry learning techniques in information technology courses. Journal of Learning Design, 6, 1-11.

Utley, C. A., \& And Others. (1997). Peer-Mediated Instruction and Interventions. Focus on Exceptional Children, 29(5), 1-23.

van Rooij, S. W., \& Zirkle, K. (2016). Balancing pedagogy, student readiness and accessibility: A case study in collaborative online course development. The Internet and Higher Education, 28, 1-7. https://doi.org/10.1016/j.iheduc.2015.08.001

Vanags, T., Pammer, K., \& Brinker, J. (2013). Process-oriented guided-inquiry learning improves long-term retention of information. Advances in Physiology Education, 37(3), 233-241. https://doi.org/10.1152/advan.00104.2012

Villagonzalo, E. C. (2014). Process oriented guided inquiry learning: An effective approach in enhancing students' academic performance. In DLSU Research congress (Vol. 2, pp. 1-6).

Yuliastini, I. B., Rahayu, S., Fajaroh, F., \& Mansour, N. (2018). Effectiveness of POGIL with SSI Context on Vocational High School Students' Chemistry Learning Motivation. Jurnal Pendidikan IPA Indonesia, 7(1), 85-95. https://doi.org/10.15294/jpii.v7i1.9928

D. |Aragon, S., \& R., S. (2002, November 30). An Instructional Strategy Framework for Online Learning Environments. Retrieved from https://eric.ed.gov/?id=EJ772939 